\title{
Driver-witness electron beam acceleration in dielectric mm-scale capillaries
}

\author{
K. Lekomtsev, ${ }^{1,}$ A. Aryshev, ${ }^{2,3}$ A. A. Tishchenko, ${ }^{4,5}$ M. Shevelev, ${ }^{6}$ A. Lyapin, ${ }^{1}$ S. Boogert, ${ }^{1}$ \\ P. Karataev, ${ }^{1}$ N. Terunuma, ${ }^{2,3}$ and J. Urakawa ${ }^{2}$ \\ ${ }^{1}$ John Adams Institute at Royal Holloway, University of London, Egham, \\ Surrey TW20 OEX, United Kingdom \\ ${ }^{2}$ KEK: High Energy Accelerator Research Organization, 1-1 Oho, Tsukuba, Ibaraki 305-0801, Japan \\ ${ }^{3}$ SOKENDAI: The Graduate University for Advanced Studies, 1-1 Oho, Tsukuba, Ibaraki 305-0801, Japan \\ ${ }^{4}$ National Research Nuclear University MEPhI, Kashirskoe Highway, \\ 31, Moscow 115409, Russian Federation \\ ${ }^{5}$ National Research Center Kurchatov Institute, Akademika Kurchatova pl. 1, \\ Moscow 123182, Russian Federation \\ ${ }^{6}$ Tomsk Polytechnic University, Institute of Physics and Technology, \\ Lenin Avenue 30, Tomsk 634050, Russian Federation
}

(Received 11 October 2017; published 10 May 2018)

\begin{abstract}
We investigated a corrugated mm-scale capillary as a compact accelerating structure in the driverwitness acceleration scheme, and suggested a methodology to measure the acceleration of the witness bunch. The accelerating fields produced by the driver bunch and the energy spread of the witness bunch in a corrugated capillary and in a capillary with a constant inner radius were measured and simulated for both on-axis and off-axis beam propagation. Our simulations predicted a change in the accelerating field structure for the corrugated capillary. Also, an approximately twofold increase of the witness bunch energy gain on the first accelerating cycle was expected for both capillaries for the off-axis beam propagation. These results were confirmed in the experiment, and the maximum measured acceleration of $170 \mathrm{keV} / \mathrm{m}$ at $20 \mathrm{pC}$ driver beam charge was achieved for off-axis beam propagation. The driver bunch showed an increase in energy spread of up to $11 \%$, depending on the capillary geometry and beam propagation, with a suppression of the longitudinal energy spread in the witness bunch of up to $15 \%$.
\end{abstract}

DOI: 10.1103/PhysRevAccelBeams.21.051301

\section{INTRODUCTION}

Dielectric wakefield acceleration (DWA) in planar and circular dielectric structures has seen major developments in understanding the maximum achievable accelerating gradients [1], dielectric breakdown limits [2] as well as applications for beam compression [3], modulation [4] and correlated energy spread compensation [5]. Extremely compact DWA-based accelerators have the potential to drive the next generation of free electron lasers and linear colliders [6,7]. In DWA schemes a high charge driver bunch excites Cherenkov radiation in a dielectric material that is reflected by metallic coating and can be used as an acceleration mechanism for a low charge witness bunch following the driver [8]. Key advantages of the DWA

\footnotetext{
*Corresponding author.

Konstantin.Lekomtsev@rhul.ac.uk

Published by the American Physical Society under the terms of the Creative Commons Attribution 4.0 International license. Further distribution of this work must maintain attribution to the author(s) and the published article's title, journal citation, and DOI.
}

compared for example to dielectric laser acceleration $[9,10]$ are: dielectric structures are typically larger, hence can be manufactured relatively easily using a variety of techniques, and both the driver and the witness bunches can be delivered by the same source and, therefore, naturally synchronized.

Theories estimating transverse and longitudinal wakefields in a dielectric-lined waveguide $[11,12]$ were developed following the experiment that demonstrated the feasibility of dielectric wakefield acceleration [8]. The longitudinal decelerating field $e E_{\mathrm{z} \text {,dec }}$ within the driver beam was estimated in [2] based on the classical problem of Cherenkov radiation in the presence of a dielectric boundary [13]:

$$
e E_{\mathrm{z}, \mathrm{dec}}=e E_{\mathrm{z}, \mathrm{surf}} \frac{\sqrt{\epsilon-1}}{\epsilon} \approx-\frac{4 N_{b} r_{e} m_{e} c^{2}}{a\left[\sqrt{\frac{8 \pi}{\epsilon-1}} \epsilon \sigma_{z}+a\right]},
$$

where $e$ is the electron charge, $a$ inner radius of the dielectric tube, $\sigma_{z} \approx c \sigma_{t}$ rms bunch length, $r_{e}$ and $m_{e} c^{2}$ the classical electron radius and rest energy of the electron, $\epsilon$ dielectric relative permittivity, and $N_{b}$ number of electrons in the bunch. In theoretical investigations $[14,15]$ a corrugated channel in a 
dielectric was considered as a source of both Cherenkov and Smith-Purcell radiation (SPR), it was shown that the intensity of radiation produced from a corrugated channel for small values of the inner radius $<1 \mathrm{~mm}$ can be an order of magnitude higher than the radiation from a channel with a constant inner radius. The Cherenkov radiation and SPR follow a dispersion relation,

$$
\cos \Theta=\frac{2 \pi m}{k d}+\frac{1}{\beta \sqrt{\epsilon_{d}(\omega)}},
$$

where $\Theta$ is the azimuthal angle with respect to the bunch velocity, $\beta$ normalized bunch velocity, $k$ wave number in the dielectric, $d$ groove period, $m$ diffraction order and $\epsilon_{d}(\omega)$ dielectric permittivity as a function of frequency. For $m=0$ one gets the dispersion relation of the Cherenkov radiation, while $m=-1,-2,-3, \ldots$ correspond to the SPR. The theory gives a clear indication that high intensity fields with a distinct frequency spectrum can be produced inside a vacuum channel once the radiation is reflected back from the waveguide cladding and diffracted at the corrugation. However, relation (2) was derived for a corrugated vacuum channel in the assumption of an infinite length dielectric, therefore limiting its direct applicability to the problem of estimating the electric field in a realistic capillary.

In this paper we describe our initial steps in understanding wakefield acceleration in a mm-scale corrugated dielectric capillary in contrast to a capillary with a constant inner radius. We discuss how the corrugation changes the frequency spectrum of the accelerating field. We compare the measurements and simulations of the witness bunch acceleration and energy spread of both witness and driver bunches in corrugated and conventional dielectric lined capillaries with the same inner radius. In the rest of the text the former will be referred to as a corrugated capillary and the latter as a flat capillary. The term $\mathrm{mm}$-scale is used to differentiate the cylindrical dielectric structures with typical transverse sizes in the order of a millimeter [16] from capillaries with aperture sizes in the order of hundreds of micrometers $[17,18]$.

\section{EXPERIMENT}

The experimental study was carried out at the laser undulator compact x-ray facility (LUCX) in High Energy Accelerator Research Organization (KEK), Japan [19]. The $\mathrm{mm}$-scale capillaries used in the experiment (see Table I) were constructed as sets of dielectric cylindrical rings mounted into copper casings and secured by copper holders on both sides.

The axial cross sections of both the corrugated and flat capillaries are shown in Fig. 1(a), a cross section of the corrugated capillary assembly with the holders is shown in Fig. 1(b). The inner radius $r_{1}$ was chosen to be $2 \mathrm{~mm}$ : wide enough to avoid the beam clipping and study the electron beam propagation with a transverse offset with respect to the capillary axis. As an approximation, a corrugation depth
TABLE I. KEK: LUCX, beam parameters at rf gun section and capillary parameters.

\begin{tabular}{lc}
\hline \hline Parameter & Value \\
\hline Driver bunch charge $Q_{\mathrm{dr}}$ & $20 \mathrm{pC}$ \\
Witness bunch charge $Q_{\mathrm{w}}$ & $\approx Q_{\mathrm{dr}} / 3$ \\
Driver bunch $\sigma_{\text {transv }}^{\mathrm{dr}}$ & $230 \mu \mathrm{m}$ \\
Witness bunch $\sigma_{\text {transv }}^{\mathrm{w}}$ & $90 \mu \mathrm{m}$ \\
Driver bunch $\sigma_{\mathrm{long}}^{\mathrm{dr}}$ & $0.5 \mathrm{ps}(0.15 \mathrm{~mm})$ \\
Witness bunch $\sigma_{\text {long }}^{\mathrm{w}}$ & $0.35 \mathrm{ps}(0.105 \mathrm{~mm})$ \\
Repetition rate, max & $3.13 \mathrm{~Hz}$ \\
Normalized emittance, typical & $1.5 \times 1.5 \mathrm{~mm} \mathrm{mrad}$ \\
Driver-to-witness separation & $1.6-3.6 \mathrm{~mm}$ \\
Capillary length, $L$ & $60 \mathrm{~mm}$ \\
Capillary material & Fused quartz \\
$r_{1} ; r_{2} ; r_{3}$ & $2 ; 2.2 ; 2.7 \mathrm{~mm}$ \\
Corrugation period, $d$ & $1 \mathrm{~mm}$ \\
\hline \hline
\end{tabular}

of $0.2 \mathrm{~mm}$ was chosen to yield the maximum of SPR intensity at $300 \mathrm{GHz}$ by analogy with a lamellar diffraction grating, which yields the maximum radiation intensity at a chosen wavelength, $\lambda$, when the depth of the grating is $\approx \lambda / 4[20,21]$. The alignment of the capillaries with respect to the electron beam was performed using the so-called "gamma scans": The beam is scanned transversely across the capillary and the bremsstrahlung radiation, produced when the beam hits the material, is detected by an air-Cherenkov detector. Where the weakest signal is detected, this corresponds to the beam propagation through the capillary aperture.

An electron beam (see Table I) was produced by illuminating a $\mathrm{Cs}_{2} \mathrm{Te}$ photocathode by an up-converted titaniumsapphire laser beam with femtosecond pulse duration. A twofold Michelson interferometer was used to produce two laser pulses that generated two electron bunches with a variable delay, directly from the photocathode. Driver and witness bunches of unequal charges were produced by introducing a laser pulse intensity imbalance [22]. The distance between the bunches was adjustable in a range of 1.6 to $3.6 \mathrm{~mm}$. The two bunches were accelerated to about
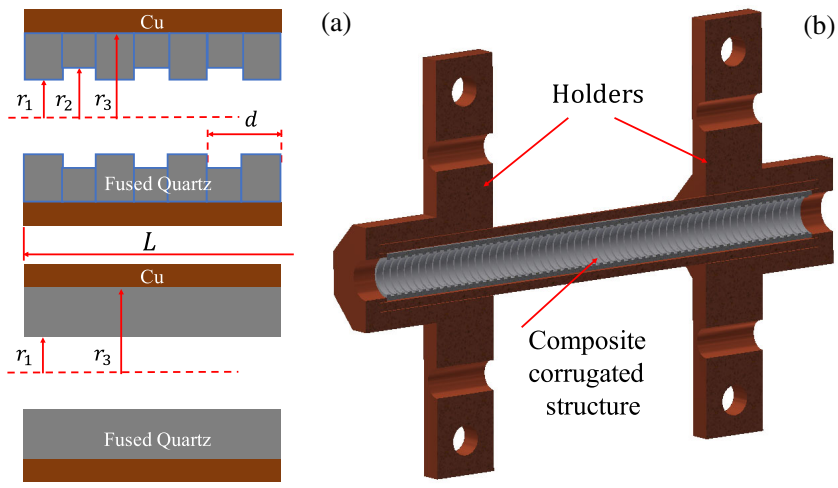

FIG. 1. Cross sections of corrugated capillary and capillary (a), cross section of corrugated mm-scale capillary with holders (b). 


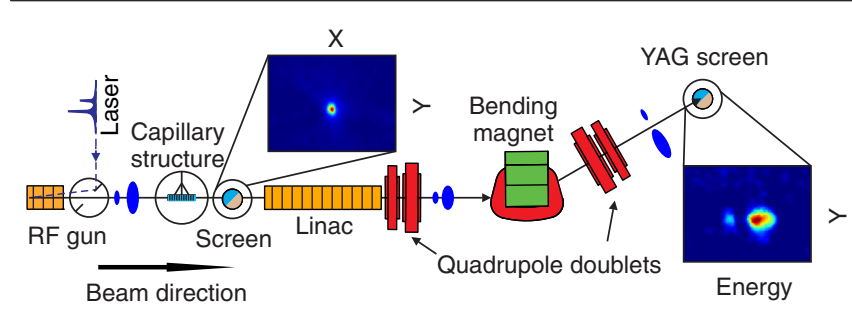

FIG. 2. Schematic view of driver-witness experiment.

$8 \mathrm{MeV}$ in a 3.6 cell S-band rf gun. A detailed explanation of this beam generation technique can be found in [19]. The rf gun introduced a time-energy correlation in the beam, thus producing driver and witness bunches of energies differing by around $80 \mathrm{keV}$. The beam was dispersed by the bending magnet (Fig. 2), so that the different time slices were projected to the different transverse coordinates on the yttrium aluminium garnet screen (YAG).

The YAG screen was placed in the location where the dispersive beam size contribution to the measured profile $\eta_{x} d E / E=990 \mu \mathrm{m}$ was larger than the betatron size contribution $\left[\beta_{x} \epsilon_{x} / \gamma\right]^{1 / 2}=180 \mu \mathrm{m}$, where $\gamma, \beta_{x}, d E / E$ and $\eta_{x}$ are the Lorentz factor, horizontal beta function, energy spread and horizontal dispersion, respectively. Energy measurements were made relative to a no structure reference measurement. Therefore, the effect of image broadening on the YAG screen neither affected the driver-to-witness measurements nor significantly influenced the accuracy of the measured energy spread. The rms resolution of the beam CCD measurement was approximately $5.5 \mathrm{keV}$.

\section{SIMULATIONS}

Simulations of the driver-witness beam interaction with the capillaries were performed using Computer Simulation
Technologies (CST) Particle Studio Particle In Cell (PIC) solver [23]. The driver and witness bunches were simulated as sets of variable weight macroparticles with a Gaussian distribution of charge both transversely and longitudinally (refer to Table I for beam parameters). The holders [Fig. 1(b)] were not taken into account in simulations, and the metallic boundary was represented as a perfect electric conductor coating. The dielectric (fused quartz) was assumed to be loss free.

Initially, in order to see the fields created by the driver bunch in the structure, the driver was simulated separately. These simulations resulted in spatial distributions of the accelerating electric field (Fig. 3). Then both the driver and the witness bunches were loaded in the domain and by using open boundary conditions the electromagnetic waves were allowed to escape the domain without reflecting back on its borders, which could affect the fields acting on the witness bunch. The driver-witness beam was loaded on axis or $1.2 \mathrm{~mm}$ off axis at the capillary entrance. The domain was extended longitudinally for the beam to fully exit the capillary. This way, the energy spread and mean energy in both the witness and driver bunches were estimated after propagation through the accelerating structures. The simulation suffered the effect of numeric Cherenkov noise caused by the mesh size limited resolution at high frequencies [24]. The mesh settings (Table II) were chosen to sufficiently resolve the longitudinal beam profile, while keeping reasonable computational time (6-8 hours on a ten-core computational node) and memory consumption (limited to $128 \mathrm{~GB}$ ). A controlled dispersion approach was used, in which the vacuum in the wave to macroparticle interaction region of the simulation domain is replaced by a material with the transmission of less than $1 \%$ for frequencies above the chosen $360 \mathrm{GHz}$ cutoff.

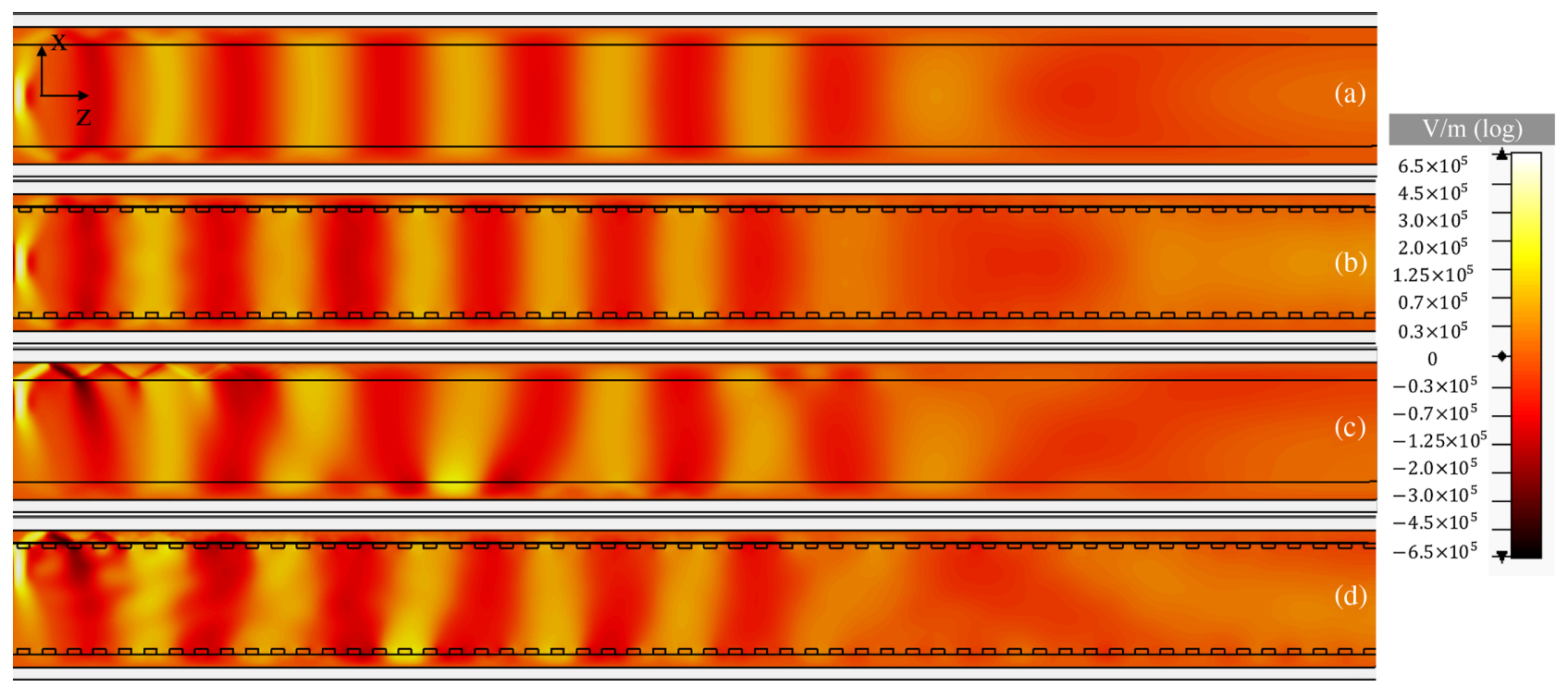

FIG. 3. Field maps for on axis [(a) and (b)] and $1.2 \mathrm{~mm}$ off axis [(c) and (d)] beam propagations in capillaries. 
TABLE II. Mesh and macroparticle parameters for second simulation setup. PBA-perfect boundary approximation.

\begin{tabular}{lc}
\hline \hline Parameter & Value \\
\hline Mesh type & Hexahedral with PBA \\
Average longitudinal size of cell & $26 \mu \mathrm{m}$ \\
Average transverse size of cell & $24 \mu \mathrm{m}$ \\
$\mathrm{N}$ of cells & 180 million \\
$\mathrm{N}$ of macroparticles in driver & 86697 \\
$\mathrm{~N}$ of macroparticle in witness & 55738 \\
\hline \hline
\end{tabular}

The accelerating fields produced by the driver bunch are plotted in Fig. 3. The field maps are shown as slices in the $z x$ plane, where $z$ coincides with the capillary axis and $x$ is the transverse direction in which the offset is introduced when the beam propagates off axis. The field maps are shown for four cases: on-axis beam propagation in the flat [Fig. 3(a)] and corrugated [Fig. 3(b)] capillary and off-axis propagation with a transverse offset of $1.2 \mathrm{~mm}$ in the flat [Fig. 3(c)] and corrugated [Fig. 3(d)] capillary. In all four cases five distinct acceleration-deceleration cycles of the $E_{z}$ field are visible before the oscillations decay almost completely. When the driver beam propagates off axis, the field pattern becomes distorted, with the maximum of the accelerating field shifting closer to the dielectric layer. In case of the corrugated capillary, the accelerating-decelerating cycles are more closely spaced. In Fig. 3 the maximum decelerating field for the case of axial beam propagation in the flat capillary is $87 \mathrm{kV} / \mathrm{m}$. The corresponding maximum decelerating field predicted by formula (1) is $97 \mathrm{kV} / \mathrm{m}$.

In order to analyze the spectrum of the observed cycles a field probe was placed at the beginning of the simulated structure on the beam axis. The accelerating field intensity of the first five acceleration-deceleration cycles was recorded as a function of time. A Fourier transformation

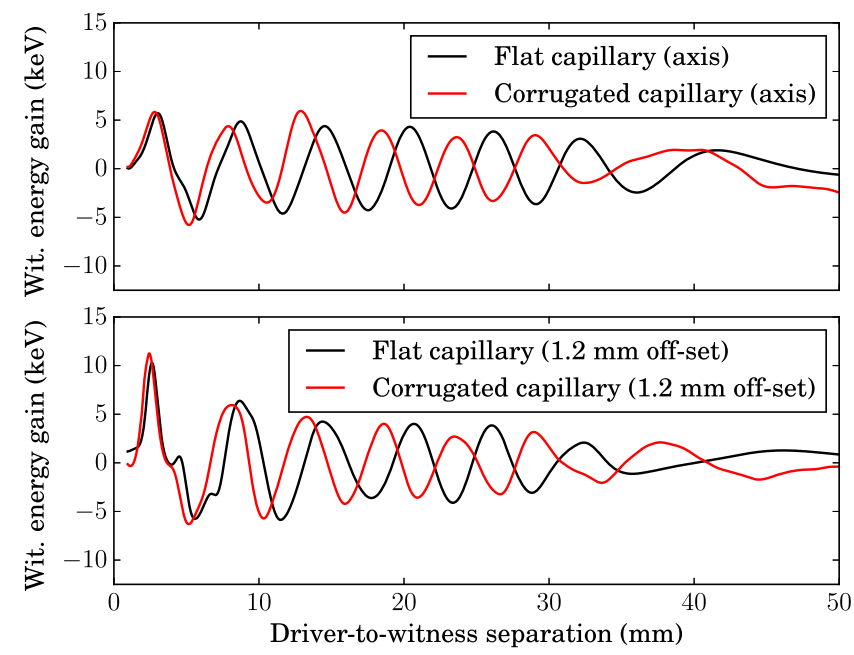

FIG. 4. Predicted on-axis and $1.2 \mathrm{~mm}$ off-axis accelerationdeceleration vs bunch separation.
TABLE III. Measured charge imbalances.

\begin{tabular}{lc}
\hline \hline Parameter & Value \\
\hline Reference measurement & $2.21 \pm 0.05$ \\
Corrugated capillary, center & $2.56 \pm 0.07$ \\
Corrugated capillary, off center & $2.55 \pm 0.03$ \\
Flat capillary, center & $2.55 \pm 0.06$ \\
Flat capillary, off center & $3.20 \pm 0.04$ \\
\hline \hline
\end{tabular}

of the time domain data showed that in the case of the corrugated capillary the frequency of the dominant accelerating mode increases to $55 \mathrm{GHz}$ compared to $50 \mathrm{GHz}$ in the case of the flat capillary. Corrugation changes the phase velocity of the wakefield, and therefore shifts the frequency of the main accelerating mode and modifies its dispersion relation, which is reported, for example, in [7].

In the experiment the witness bunch was moving colinearly to the driver beam in both on-axis and off-axis regimes. The accelerating field along the witness bunch trajectory was integrated resulting in the bunch energy gain, Fig. 4, in the assumption that the distance between the bunches does not change over the length of the structure.

In two-beam simulations, the ratio of driver to witness bunch charges was set in accordance to the average intensity imbalance between the bunches measured experimentally with the YAG screen (Table III). The distance between the bunches for each case was set to the peak of the acceleration, as determined in the case when only the drive beam is present. The energy of each macroparticle was recorded in order to calculate the standard deviation of the energy distribution of both the driver and witness bunches.

The fractional relative decrease of the energy spread in the witness bunch was calculated using

$$
\frac{\Delta \sigma_{\mathrm{W}}}{\sigma_{\mathrm{W}}}=\frac{\sigma_{W_{\text {reference }}}-\sigma_{W_{\text {capillary }}}}{\sigma_{W_{\text {reference }}}},
$$

where $\sigma_{W_{\text {reference }}}$ is the witness energy spread in the reference simulation that assumed the driver-witness pair propagation in free space (still filled with the dispersive material as described above). Similarly, the fractional relative increase of the energy spread in the driver bunch was calculated as

$$
\frac{\Delta \sigma_{\mathrm{D}}}{\sigma_{\mathrm{D}}}=\frac{\sigma_{D_{\text {capillary }}}-\sigma_{D_{\text {reference }}}}{\sigma_{D_{\text {reference }}}},
$$

where $\sigma_{D_{\text {reference }}}$ is the driver energy spread in the reference simulation.

Simulated results are presented together with the experimental ones in the following section.

\section{RESULTS AND DISCUSSION}

During the experiment the energy distribution in the driver-witness beam was measured as a function of the bunch-to-bunch separation. The bunch-to-bunch separation 


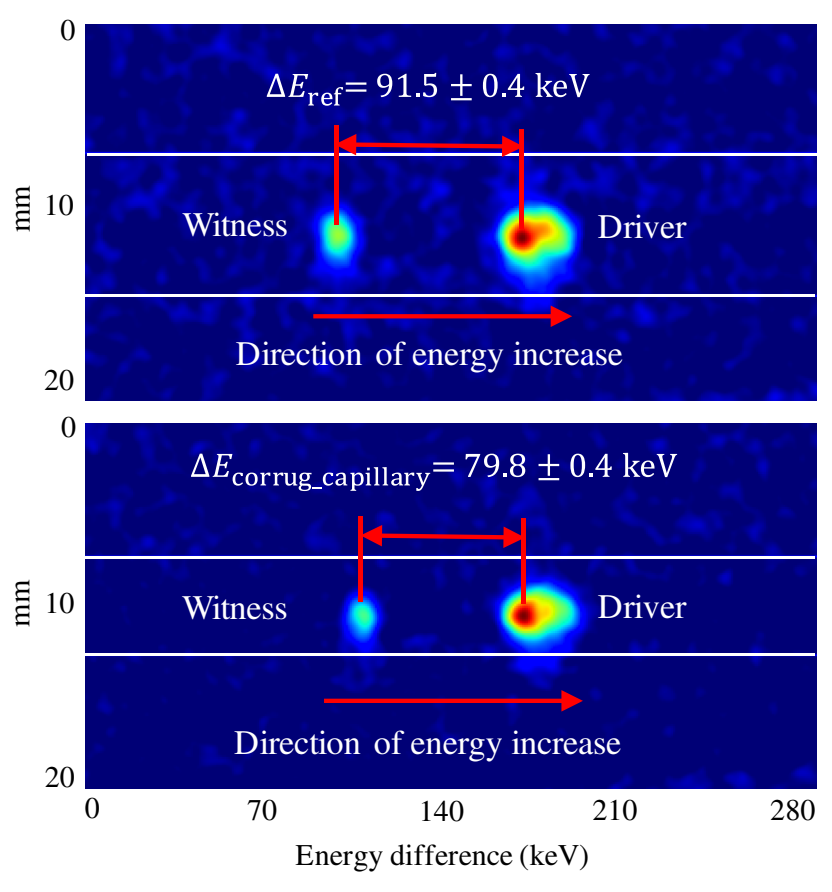

FIG. 5. CCD image of YAG screen.

was continuously scanned, while CCD images from the YAG screen were recorded.

To obtain the energy difference between the driver and the witness, each image was projected in the vertical direction within a narrow vertical region of interest (white lines in Fig. 5) and fitted with a skewed-double-Gaussian function [see Eq. (5)] to provide information about the energy distribution in the driver bunch.

$$
\begin{aligned}
& f\left(x, \mu_{\mathrm{w}}, \sigma_{\mathrm{w}}, \alpha_{\mathrm{w}}, \mu_{\mathrm{dr}}, \sigma_{\mathrm{dr}}, \alpha_{\mathrm{dr}}\right) \\
& =\frac{1+\operatorname{erf}\left(\frac{\alpha_{\mathrm{w}}\left(x-\mu_{\mathrm{w}}\right)}{\sqrt{2}}\right)}{\sqrt{2 \pi} \sigma_{\mathrm{w}}} \times \exp \left(-\frac{\left(x-\mu_{\mathrm{w}}\right)^{2}}{2 \sigma_{\mathrm{w}}^{2}}\right) \\
& \quad+\frac{1+e r f\left(\frac{\alpha_{\mathrm{dr}}\left(x-\mu_{\mathrm{dr}}\right)}{\sqrt{2}}\right)}{\sqrt{2 \pi} \sigma_{\mathrm{dr}}} \times \exp \left(-\frac{\left(x-\mu_{\mathrm{dr}}\right)^{2}}{2 \sigma_{\mathrm{dr}}^{2}}\right),
\end{aligned}
$$

where $x$ is a horizontal coordinate, $\mu_{\mathrm{w}}, \sigma_{\mathrm{w}}, \alpha_{\mathrm{w}}$ are mean, standard deviation and skewness parameter for the energy distribution of the witness beam, and $\mu_{\mathrm{dr}}, \sigma_{\mathrm{dr}}, \alpha_{\mathrm{dr}}$ are the same parameters but for the driver beam, $\operatorname{erf}(\cdots)$ is the error function.

Pixel units were then converted into the distance on the screen, using the calibration curve of the CCD optical system. For each bunch-to-bunch distance the difference between the centroids on the YAG screen was binned and the mean and rms error computed. As a final step, the energy difference in terms of the distance on screen was converted into the units of energy using the distance-energy calibration (Fig. 6).

The measure of the witness bunch acceleration was the relative energy difference between the driver and the

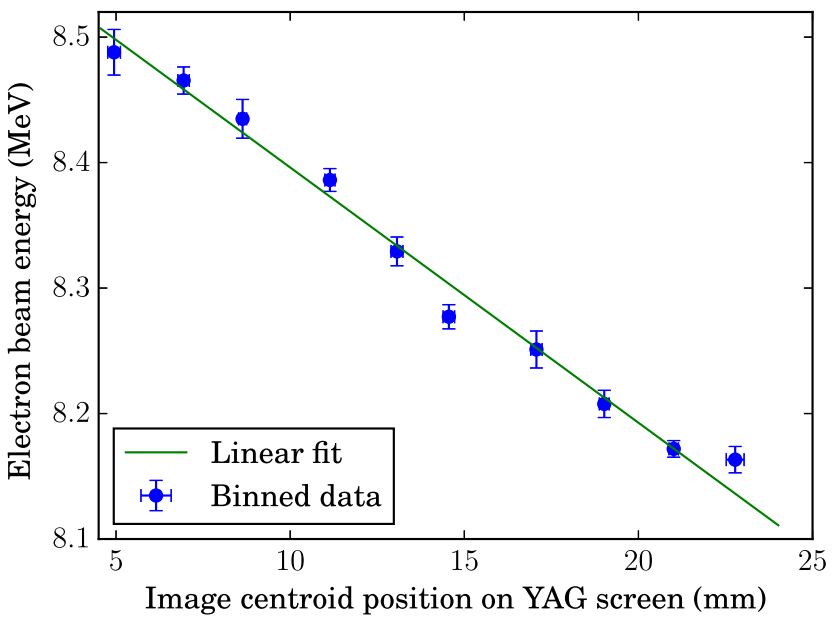

FIG. 6. Bending magnet energy calibration. Linear fit: $A+B x$, $A=8.60 \pm 0.01[\mathrm{MeV}], B=-0.020 \pm 0.001[\mathrm{MeV} / \mathrm{mm}]$.

witness with respect to the reference measurement, which was performed when the beam propagated in the vacuum chamber without a capillary in its path. $\Delta E_{\text {reference }}-$ $\Delta E_{\text {capillary }}$ gives the acceleration of the witness bunch as a function of the bunch-to-bunch distance (Fig. 7). Where the value of the energy difference on the YAG screen $\Delta E_{\text {capillary }}<\Delta E_{\text {reference, }}$, we observe acceleration (see Fig. 5 for two typical CCD images), while $\Delta E_{\text {capillary }}>\Delta E_{\text {reference }}$ corresponds to deceleration.

The scanning range of the bunch-to-bunch separation of 1.6-3.6 $\mathrm{mm}$ was chosen to match the region of the linear growth of the driver-witness energy difference in the reference measurement. The electron beam charge stability was within $0.3 \%$ for all measurements. Measured acceleration dependencies were compared to the simulations of the first acceleration cycle, shown by green fillings in Figs. 7(a) and 7(b).

There is a good agreement between the measurements and simulations in terms of the maximum achievable acceleration (Table IV) both for the flat capillary [Figs. 7(c) and 7(d)] and the corrugated capillary [Figs. 7(e) and 7(f)]. The simulations predict that in the case of the corrugated capillary accelerating cycles become more closely spaced, however this effect is small for the driver-to-witness separation region where the experimental data were obtained. It is more prominently visible in the case of the off-axis beam propagation in the corrugated capillary [Fig. 7(f)].

We also studied the change in the energy spread at the maxima of acceleration. The standard deviation of the longitudinal energy spread was averaged over narrow regions of the bunch-to-bunch separation for the experimental measurements and simulations, shown in Fig. 7 by vertical dashed lines. To obtain average simulated values, in each case ten separate PIC simulations were performed for ten equidistant bunch-to-bunch separations in the marked regions. Results are presented in Table V. The measured values of the witness bunch energy spread suppression are 

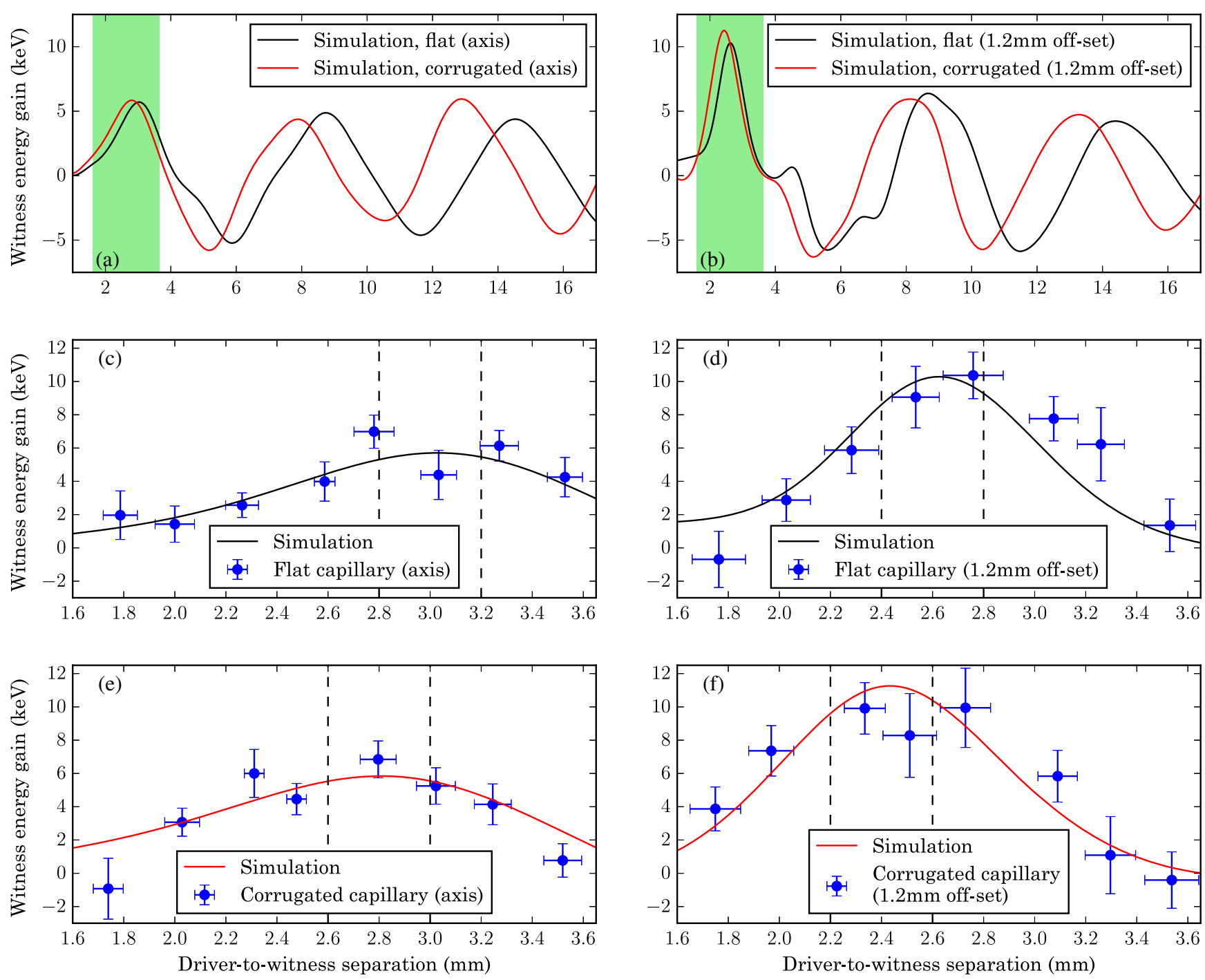

FIG. 7. First three acceleration-deceleration cycles [(a) and (b)], from Fig. 4, and comparison with experimental data for on-axis [(c) and (e)] and $1.2 \mathrm{~mm}$ off-axis [(d) and (f)] beam propagation in capillaries.

in a wide statistical region of $3.5 \%-32 \%$; the simulations indicate a narrower region of $13.7 \%-15.5 \%$. For the driver bunch the measured values of the energy spread increase are in the region of $0 \%-16.8 \%$; the simulated values are in the region of $3.7 \%-11.2 \%$. Considering an intersection of the simulated and the experimental ranges, we may conclude that the driver bunch showed an increase in the energy spread of up to $11 \%$, the witness bunch showed a suppression of the longitudinal energy spread of up to $15 \%$, for the given experimental conditions.

Additionally, we observed a high energy leading edge in the driver bunch (Fig. 5), which was clearly present in the majority of the recorded images. The average skewness of the energy distribution of the driver bunch was calculated to ensure a correct interpretation of the relative energy gain of the witness bunch. The reference measurement had

TABLE IV. Maxima of witness bunch acceleration.

\begin{tabular}{lccccc}
\hline \hline & \multicolumn{2}{c}{ Witness max acceleration, keV } & & \multicolumn{2}{c}{ Driver-to-witness separation, mm } \\
\cline { 2 - 3 } Geometry & \multicolumn{1}{c}{ Measured } & Simulated & & Measured & Simulated \\
\hline Corrugated capillary, axis & $6.85 \pm 1.11$ & 5.84 & & $2.80 \pm 0.07$ & 2.81 \\
Corrugated capillary, off axis & $9.91 \pm 1.55$ & 11.26 & & $2.33 \pm 0.08$ & 2.43 \\
Flat capillary, axis & $4.39 \pm 1.48$ & 5.71 & & $3.03 \pm 0.07$ & 3.02 \\
Flat capillary, off axis & $10.37 \pm 1.40$ & 10.29 & & $2.76 \pm 0.12$ & 2.63 \\
\hline \hline
\end{tabular}


TABLE V. Relative energy spread in witness and driver bunches calculated by Eqs. (3) and (4).

\begin{tabular}{lccrrr}
\hline \hline & \multicolumn{2}{c}{$\frac{\Delta \sigma_{W}}{\sigma_{W}}, \%$} & & $\frac{\Delta \sigma_{D}}{\sigma_{D}}, \%$ \\
\cline { 2 - 3 } Geometry & Measured & Simulated & & Measured & Simulated \\
\hline Corrugated capillary, axis & $22.0 \pm 7.9$ & 13.7 & $4.9 \pm 4.8$ & 3.8 \\
Corrugated capillary, off axis & $9.2 \pm 5.7$ & 14.5 & $12.2 \pm 4.6$ & 11.2 \\
Flat capillary, axis & $19.8 \pm 12.2$ & 15.3 & $4.6 \pm 4.6$ & 3.7 \\
Flat capillary, off axis & $13.2 \pm 6.8$ & 15.5 & $5.1 \pm 4.8$ & 11.1 \\
\hline \hline
\end{tabular}

an average skewness of the driver bunch of $\alpha_{\mathrm{dr}}=0.588 \pm$ 0.233 . The average value of the driver beam skewness did not change significantly with introduction of the structures, and was, in fact, less or equal to the value obtained in the reference measurement. Consequently, the core of the driver bunch did not experience deceleration with respect to its head. Considering reasonable agreement between the measured and simulated acceleration dependencies, one can argue that any changes in the energy difference between the driver and the witness bunches come from the acceleration or deceleration of the witness bunch.

\section{CONCLUSIONS}

We compared performance of the corrugated and the flat capillary as accelerating structures in the driver-witness acceleration scheme and as devices capable to reduce energy spread of the witness bunch. The maximum acceleration of $170 \mathrm{keV} / \mathrm{m}$ at $20 \mathrm{pC}$ driver bunch charge was measured for the beam propagation with a transverse offset in both capillaries, assuming that the energy gain increases linearly with the number of periods for the corrugated capillary. An approximately twofold increase in the witness bunch energy gain on the first accelerating cycle for the offaxis beam propagation was confirmed by the experiment and simulations. Both the corrugated and flat capillaries demonstrated similar performance in reducing the energy spread of the witness bunch, while the measured and simulated energy spread of the driver bunch were only moderately higher for the corrugated capillary. We should point out that the corrugated beam pipe walls [25] and the corrugated planar structures with orthogonal orientations [26] were also reported to significantly reduce energy spread in relativistic electron bunches.

Both the corrugated and flat capillaries are capable of supporting multiple modes due to a relatively large thickness of the dielectric layer compared to the inner radius of the capillaries [7]. We are currently considering to extend the method of polarization current density $[14,27]$ with the addition of the mode analysis. It would allow a comprehensive description of the fields acting on the electron beam and ensure better coupling between the two analytical approaches.

We showed that the corrugation changed the phase velocity of the wakefield and shifted the frequency of the main accelerating mode from 50 to $55 \mathrm{GHz}$. This effect was more prominently visible for off-axis beam propagation. For the chosen corrugation depth and period there was no measurable increase in the accelerating field compared to the flat capillary. An optimization of the corrugated capillary parameters is required to explore its capability to provide accelerating gradients higher than in the flat capillary. The linear scaling of the accelerating gradient as a function of the driver bunch charge was confirmed by PIC simulations. However, with introduction of the corrugation, the dependencies of the accelerating gradient on the capillary radius, dielectric constant [see Eq. (1)] and thickness of the dielectric layer are modified, and there are additional parameters such as the corrugation depth and period as well as the number of periods. There is also a question of synchronicity of the accelerating field and the witness bunch for longer accelerating structures. This requires both extensive analytical and numerical modeling of corrugated capillaries. Further speculation leads to modifications to the metallic boundary and self-similar fractal corrugations of the dielectric engaging higher spatial harmonics of the field in acceleration.

\section{ACKNOWLEDGMENTS}

This project has received funding from the European Union Horizon 2020 research and innovation program under the Marie Sklodowska-Curie Grant Agreement No. 655179. This work was supported by the Science Technology Facilities Council (Grants No. ST/J002038/1 and No. ST/P00203X/1). Additionally, A. A. Tishchenko is thankful for support to the Leverhulme Trust International Network, Grant No. IN-2015-012, as well as the Ministry of Science and Education of the Russian Federation, Grant No. 3.2621.2017/4.6, and the Competitiveness Programme of National Research Nuclear University "MEPhI." M. Shevelev's contribution was supported by the Federal Targeted Program of the Russian Federation Agreement No. 14.578.21.0198 (IDN RFMEFI57816X0198) and by the Competitiveness enhancement program of Tomsk Polytechnic University.

[1] B. O’Shea, G. Andonian, S. Barber, K. Fitzmorris, S. Hakimi, J. Harrison, P. Hoang, M. Hogan, B. Naranjo, O. Williams, V. Yakimenko and J. B. Rosenzweig, Observation 
of acceleration and deceleration in gigaelectron-voltper-metre gradient dielectric wakefield accelerators, Nat. Commun. 7, 12763 (2016).

[2] M. Thompson, H. Badakov, A. Cook, J. Rosenzweig, R. Tikhoplav, G. Travish, I. Blumenfeld, M. Hogan, R. Ischebeck, N. Kirby et al., Breakdown Limits on Gigavolt-per-Meter Electron-Beam-Driven Wakefields in Dielectric Structures, Phys. Rev. Lett. 100, 214801 (2008).

[3] L. J. Wong, A. Fallahi, and F. X. Kärtner, Compact electron acceleration and bunch compression in $\mathrm{THz}$ waveguides, Opt. Express 21, 9792 (2013).

[4] S. Antipov, C. Jing, M. Fedurin, W. Gai, A. Kanareykin, K. Kusche, P. Schoessow, V. Yakimenko, and A. Zholents, Experimental Observation of Energy Modulation in Electron Beams Passing through Terahertz Dielectric Wakefield Structures, Phys. Rev. Lett. 108, 144801 (2012).

[5] Y. Nie, R. Assmann, U. Dorda, B. Marchetti, M. Weikum, J. Zhu, and M. Hüning, Potential applications of the dielectric wakefield accelerators in the SINBAD facility at DESY, Nucl. Instrum. Methods Phys. Res., Sect. A 829, 183 (2016).

[6] E. A. Nanni, W. R. Huang, K.-H. Hong, K. Ravi, A. Fallahi, G. Moriena, R. D. Miller, and F. X. Kärtner, Terahertz-driven linear electron acceleration, Nat. Commun. 6, 8486 (2015).

[7] C. Jing, Dielectric wakefield accelerators, Rev. Accel. Sci. Techol. 09, 127 (2016).

[8] W. Gai, P. Schoessow, B. Cole, R. Konecny, J. Norem, J. Rosenzweig, and J. Simpson, Experimental Demonstration of Wake-Field Effects in Dielectric Structures, Phys. Rev. Lett. 61, 2756 (1988).

[9] R. J. England, R. J. Noble, K. Bane, D. H. Dowell, C.-K. Ng, J. E. Spencer, S. Tantawi, Z. Wu, R. L. Byer, E. Peralta et al., Dielectric laser accelerators, Rev. Mod. Phys. 86, 1337 (2014).

[10] E. Peralta, K. Soong, R. England, E. Colby, Z. Wu, B. Montazeri, C. McGuinness, J. McNeur, K. Leedle, D. Walz et al., Demonstration of electron acceleration in a laser-driven dielectric microstructure, Nature (London) 503, 91 (2013).

[11] M. Rosing and W. Gai, Longitudinal- and transverse-wakefield effects in dielectric structures, Phys. Rev. D 42, 1829 (1990).

[12] K.-Y. Ng, Wake fields in a dielectric-lined waveguide, Phys. Rev. D 42, 1819 (1990).

[13] B. M. Bolotovskii, Theory of Cerenkov radiation (III), Phys.-Usp. 4, 781 (1962).

[14] A. A. Ponomarenko, M. I. Ryazanov, M. N. Strikhanov, and A. A. Tishchenko, Terahertz radiation from electrons moving through a waveguide with variable radius, based on Smith-Purcell and Cherenkov mechanisms, Nucl. Instrum. Methods Phys. Res., Sect. B 309, 223 (2013).

[15] A. A. Ponomarenko, A. A. Tishchenko, and M. N. Strikhanov, $\mathrm{THz}$ polarization radiation from electrons passing corrugated dielectric tube under non-central propagation, Nucl. Instrum. Methods Phys. Res., Sect. B 402, 198 (2017).

[16] C. Jing, A. Kanareykin, J. G. Power, M. Conde, W. Liu, S. Antipov, P. Schoessow, and W. Gai, Experimental Demonstration of Wakefield Acceleration in a Tunable Dielectric Loaded Accelerating Structure, Phys. Rev. Lett. 106, 164802 (2011).

[17] S. Antipov, C. Jing, M. Fedurin, W. Gai, A. Kanareykin, K. Kusche, P. Schoessow, V. Yakimenko, and A. Zholents, Experimental Observation of Energy Modulation in Electron Beams Passing through Terahertz Dielectric Wakefield Structures, Phys. Rev. Lett. 108, 144801 (2012).

[18] G. Andonian, S. Barber, F. H. O'Shea, M. Fedurin, K. Kusche, C. Swinson, and J. B. Rosenzweig, Generation of Ramped Current Profiles in Relativistic Electron Beams Using Wakefields in Dielectric Structures, Phys. Rev. Lett. 118, 054802 (2017).

[19] A. Aryshev, M. Shevelev, Y. Honda, N. Terunuma, and J. Urakawa, Femtosecond response time measurements of a $\mathrm{Cs}_{2} \mathrm{Te}$ photocathode, Appl. Phys. Lett. 111, 033508 (2017).

[20] Y. Shibata, S. Hasebe, K. Ishi, S. Ono, M. Ikezawa, T. Nakazato, M. Oyamada, S. Urasawa, T. Takahashi, T. Matsuyama et al., Coherent Smith-Purcell radiation in the millimeter-wave region from a short-bunch beam of relativistic electrons, Phys. Rev. E 57, 1061 (1998).

[21] K. Lekomtsev, P. Karataev, A. Tishchenko, and J. Urakawa, CST simulations of $\mathrm{THz}$ Smith-Purcell radiation from a lamellar grating with vacuum gaps, Nucl. Instrum. Methods Phys. Res., Sect. B 355, 164 (2015).

[22] M. Shevelev, A. Aryshev, N. Terunuma, and J. Urakawa, Generation of a femtosecond electron microbunch train from a photocathode using twofold Michelson interferometer, Phys. Rev. Accel. Beams 20, 103401 (2017).

[23] CST PIC solver description, https://www.cst.com/products/ cstps/solvers/particle-in-cell-solver.

[24] A. D. Greenwood, K. L. Cartwright, J. W. Luginsland, and E. A. Baca, On the elimination of numerical Cerenkov radiation in PIC simulations, J. Comput. Phys. 201, 665 (2004).

[25] P. Emma, M. Venturini, K. Bane, G. Stupakov, H.-S. Kang, M. Chae, J. Hong, C.-K. Min, H. Yang, T. Ha et al., Experimental Demonstration of Energy-Chirp Control in Relativistic Electron Bunches Using a Corrugated Pipe, Phys. Rev. Lett. 112, 034801 (2014).

[26] F. Fu, R. Wang, P. Zhu, L. Zhao, T. Jiang, C. Lu, S. Liu, L. Shi, L. Yan, H. Deng et al., Demonstration of NonlinearEnergy-Spread Compensation in Relativistic Electron Bunches with Corrugated Structures, Phys. Rev. Lett. 114, 114801 (2015).

[27] A. P. Potylitsyn, M. I. Ryazanov, M. N. Strikhanov, and A. A. Tishchenko, Diffraction Radiation from Relativistic Particles, Springer Tracts in Modern Physics (Springer, Berlin, 2011), Vol. 239. 